\title{
Uses of Bile Salts, Namely Cholate and Deoxycholate for Earwax Removal
}

\author{
Craig G. Burkhart ${ }^{*}, 1$, Vijay Adappa ${ }^{2}$ and Craig N. Burkhart ${ }^{3}$ \\ ${ }^{1}$ University of Toledo College of Medicine, USA \\ ${ }^{2}$ Otolaryngology, St. Vincent's Mercy and St. Charles Hospitals, USA \\ ${ }^{3}$ Department of Dermatology, University of North Carolina at Chapel Hill, Chapel Hill, North Carolina, USA
}

\begin{abstract}
Detergents are a viable method for earwax removal. Specifically, bile salts such as cholate and deoxycholate, work well for this function especially when placed in alkaline solution provided by sodium phosphate in the presence of $0.1 \mathrm{M}$ sodium chloride. In vitro studies reveal these agents to quickly penetrate earwax, as visualized by yellowish color of the solution and lack of cohesion of the remaining particulate matter. In clinical studies, after a 5 minute application of the bile acids into ten patients' ears with impacted cerumen, only water irrigation was needed for visibility of the tympanic membrane. Further studies of cholate and dexoxycholate for ear plugs are warranted.
\end{abstract}

Cerumen serves as a protectant from bacteria and fungus as well as a cleansing and lubricating agent for the external auditory canal. Traditionally dermatology has been at the forefront in the study of earwax $[1,2]$. Impacted cerumen in the external ear canal not only interferes with the clinician's view of the tymphanic membrane, but occasionally can be associated with hearing loss, vertigo, itching, pain, tinnitus, and increased risk of infection. Ceruminous plugs are a common problem. Indeed, 2.3 million people require management of this problem in the United States. It is estimated that ear syringing is performed 4 million times annually [3]. Manual instrumentation with a curette or frequent copious syringe irrigation are the two most common methods of evacuation of cerumen plugs. Of note, these procedures can have complications which include otitis externa, hearing loss, and perforation of the tympanic membrane [4].

Cerumen plugs are caused by a constellation of factors including type of earwax, rate of cerumen accumulation, anatomic factors, and presence of excess epidermal sloughing with desquamated sheets of keratinocytes. Impacted cerumen are composed of a combination of desquamated epidermal cells lining the ear canal, hair, and secretions of the ceruminal and sebaceous glands located in the outer third of the external ear canal. The chemical composition of earwax consists of long chain fatty acids and alcohols, cholesterol precursors, squalene, and other long-chained hydrocarbons [5].

Despite limited publications on point, some detergents have ability to partially dissolve earwax. Most impressive results have been obtained with detergents which have a steroid skeleton structure, such as primary and secondary bile acids. In vitro testing has shown that bile acids, such as cholate and deoxycholate, in the appropriate mixture significantly disintegrate earwax. An analysis by flash pyroly-

*Address correspondence to this author at the University of Toledo School of Medicine, 5600 Monroe Street, Suite 106B, Sylvania, OH 43560, USA; Tel: 419-885-3403; Fax: 419-885-3401; E-mail: cgbakb@aol.com sis/gas chromatography-mass spectrometry of earwax fractionated by means of cholate and deoxycholate salts has been previously reported of our laboratory formulation [6].

For maximum dispersal of earwax, these bile salts are placed in alkaline solution provided by sodium phosphate in the presence of $0.1 \mathrm{M}$ sodium chloride. Of note, the concentration of cations significantly affects anionic detergents. These bile salts with the steroid skeleton structure, attach themselves along hydrophobic areas, thus exposing its hydrophilic tail, which pulls hydrophobic particles into solution. The dissolving of lipids with cholate and deoxycholate is by micelle formation. These bile acids are also able to surround hydrophobic parts of membrane-bound protein and move them into solution. In vitro studies reveal that these bile acids quickly penetrate earwax and lead to reduced cohesion of the particulate matter and yellowish discoloration of the solution within minutes. The current study was designed to evaluate the ceruminolytic effects in vivo of a single brief application of deoxycholate. The visibility of the tympanic membrane after the solution was drained from the canal was the primary measure of efficacy.

The project was approved by the local institutional review board. The trial was an open-label, non-blinded study in which 10 patients were selected from the office of Vijay Adappa, MD to use this formulation of deoxycholate. The patients selected were 5 males and 5 non-fertile females, and they were between the ages of 20 and 80 . All the chosen patients suffered from chronic, bilateral ceruminous plugs and on presentation had partially or completely obscured tympanic membranes. These persons were without perforated eardrums or any eczematous changes in their ear canals. All patients had previously failed with triethanolamine polypeptide (Cerumenex), and had required procedural removal of earwax.

The physician applied $1 \mathrm{~mL}$ of the study solution in each ear sequentially. The patient was instructed alternatively to lay on his/her side with the affected ear facing upward for 5 minutes. The ceruminolytic agent was allowed to drain by 
gravity into the ear canal, after which the physician irrigated the external ear canal with $50 \mathrm{~mL}$ of lukewarm normal saline solution. Then he determined whether the tympanic membrane was obscured, partially obscured, or completely visualized. After one side was completed, then the other ear was positioned for the same process and evaluation.

In all ten patients, the ear plugs were sufficiently loosened and disintegrated so that complete tympanic membrane visualization was achieved.

Several patients stated a mild drying effect with the treatment. Two patients experienced minor itching. No patient encountered any pain, vertigo, nausea, or hearing loss during the procedures. The ENT physician who administered the testing felt that the deoxycholate was distinctly superior to his prior use of triethanolamine polypeptide (Cerumenex) in this small patient sample. A prospective, double-blinded, randomized controlled trial appears warranted.

\section{REFERENCES}

[1] Akobjanoff L, Carruthers C, Senturia B. The chemistry of cerumen. J Invest Dermatol 1954; 23: 43-50.

[2] Bortz JT, Wertz PW, Downing DT. Composition of cerumen lipids J Am Acad Dermatol 1990; 23: 845-9.

[3] Guest JF, Greener MJ, Robinson AC. Impacted cerumen: composition, production, epidemiology and managements. Q J Med 2004; 97: 477-88.

[4] Bapa U, Nia J, Bance M. Severe audiovestibular loss following ear syringing for wax removal. J Laryngol Otol 2001; 115: 410-11.

[5] Burkhart CN, Burkhart CG, Williams S, Andrews PC, Adappa V, Arbogast J. In pursuit of ceruminolytic agents: a study of earwax composition. Am J Otol 2000; 21: 157-60.

[6] Burkhart CN, Kruge M, Burkhart CG, Black C. Cerumen composition by flash pyrolysis-gas chromotography/mass spectrometry. Otol Neurotol 2001; 22: 715-22. 